\title{
Pre-Exposure Prophylaxis and the Promise of Combination Prevention Approaches
}

\author{
Susan P. Buchbinder • Albert Liu
}

Published online: 18 February 2011

(c) The Author(s) 2011. This article is published with open access at Springerlink.com

\begin{abstract}
Pre-exposure prophylaxis (PrEP) for HIV prevention is a promising experimental approach currently being tested globally. A number of PrEP trials are evaluating the safety and effectiveness of PrEP in men who have sex with men (MSM) and other populations at risk for HIV, and results will be available from this first generation of efficacy trials over the next few years. Here we review the rationale for orally-administered antiretrovirals for prevention, and outline issues the first generation trials will address as well as questions that may be addressed in future studies. We also describe the rationale for combination prevention approaches that may combine PrEP with other prevention modalities as part of a larger prevention package.
\end{abstract}

Keywords Pre-exposure prophylaxis - Clinical trials · HIV prevention - Combination prevention

\section{Introduction}

"Can a pill a day prevent HIV?" That is the tagline of recruitment advertisements for an HIV prevention trial to test whether a combination pill (tenofovir/emtricitabine or Truvada $^{\circledR}$ ) can reduce the number of HIV infections when taken daily by men who have sex with men (MSM). This

S. P. Buchbinder $(\bowtie) \cdot$ A. Liu

HIV Research Section, San Francisco Department of Public

Health, 25 Van Ness Avenue, Suite 500, San Francisco,

CA 94102-6033, USA

e-mail: susan.buchbinder@sfdph.org

S. P. Buchbinder · A. Liu

University of California, San Francisco, USA approach, also known as pre-exposure prophylaxis (PrEP), is being evaluated in a number of studies in different populations worldwide, using either a daily tenofovir or Truvada ${ }^{\circledR}$ pill. Early data from PrEP trials were presented at the International AIDS Society conference in Vienna in July 2010 [1-3]; additional results are likely to be presented and/or published prior to this article appearing in print. Tenofovir gel is also being evaluated as "topical PrEP" (also known as microbicides) vaginally and rectally, and is more fully described in the article by McGowan in this issue of AIDS and Behavior.

In this article, we will review the rationale for using orally-administered antiretrovirals for prevention in HIV negative persons, both as post-exposure prophylaxis (PEP) and pre-exposure prophylaxis (PrEP). We will outline the issues the first generation of trials are designed to address as well as questions that must be addressed by future studies. Because pills alone will never completely control the AIDS epidemic, we will also discuss the rationale for combination prevention approaches that may combine PrEP (if proven efficacious) with other prevention strategies.

\section{Rationale for HIV Prophylaxis Using Antiretroviral Agents}

A number of infectious diseases can be prevented by administering antimicrobials to persons at high-risk of acquiring the disease prior to and during periods of exposure. For example, persons traveling in regions of the world with high rates of malaria take anti-malarial agents to prevent infection [4]. Antiretroviral agents have greatly reduced HIV transmission to newborns when provided to HIV-positive mothers at the time of delivery [5]. 
The first demonstration that antiretrovirals could be used to prevent HIV infection came from studies in the early 1990's of zidovudine prophylaxis in mice [6] and nonhuman primates [7]. These and later studies demonstrated that antiretrovirals were most efficacious if given prior to or within hours after HIV or SIV challenge, and continued for 4 weeks. Tsai [8] later demonstrated the improved efficacy of PMPA (tenofovir) over zidovudine in protecting adult macaques against intravenous SIV challenge. More recent studies have demonstrated the efficacy of tenofovir or Truvada ${ }^{\circledR}$ in preventing infections when animals are challenged rectally with SIV [9].

These studies, in fact, served as a proof of principle for post-exposure prophylaxis (PEP), that is, administering antiretroviral agents within hours after an HIV exposure, and continuing treatment for 4 weeks. Although no randomized controlled trial of PEP has been conducted in humans, an observational case-control study in health care workers with occupational exposures demonstrated a significantly lower rate of HIV infection among those who took AZT compared to those who did not [10], leading to the US Centers for Disease Control and Prevention's recommendations for PEP for occupational exposures [11], and eventually for high-risk non-occupational exposures [12].

Animal data suggest that for PEP to be effective, persons at risk would have to recognize they had been potentially exposed to HIV and initiate treatment within hours of exposure, making PEP an unlikely strategy for reducing HIV incidence in populations with repeated highrisk exposures. In fact, a number of PEP failures occurred in MSM who were given antiretrovirals to keep at home, but who did not initiate PEP because they did not recognize exposures as high risk [13]. A prospective study of highrisk uninfected MSM from six US cities found that only a small fraction had single exposures to HIV prior to their infection; almost all had repeated exposures, and few recognized the frequency of exposures [14]. This would suggest that, to be effective, antiretroviral agents might need to be administered throughout periods of risk, the rationale behind PrEP.

\section{PrEP Trial Overview}

The first generation of PrEP trials are projected to enroll a diverse population of more than 20,000 men and women in Africa, the Americas, and Asia (Table 1). Three PrEP studies to date have focused on MSM populations: a safety study of daily oral tenofovir in US MSM; an efficacy study of oral daily Truvada ${ }^{\circledR}$ in MSM in Peru, Ecuador, Brazil, the US, South Africa, and Thailand; and a study of PrEP safety, feasibility, and acceptability in young MSM. Other PrEP trials are exploring the safety and efficacy of tenofovir-based regimens in injection drug users (IDU), heterosexual men and women, or heterosexual couples. A website with frequently updated information about clinical PrEP trials is hosted by the AIDS Vaccine Advocacy Coalition: http://www.prepwatch.org.

Tenofovir-based regimens were chosen on the strength of pre-clinical trials, as well as the excellent safety, tolerability, and resistance profiles of these regimens when used for HIV treatment. Each of these trials will provide substantial tolerability and safety data in these diverse populations. Some trials are conducting sub-studies to assess specific safety concerns, such as additional laboratory assays to evaluate potential renal toxicity, or dual energy X-ray absorptiometry (DEXA) to evaluate the effect of tenofovir-based regimens on bone mineral density and body composition.

All studies will measure pill-taking practices through participant self-report; many studies will augment these studies through objective measures (e.g., counting returned pills, microchips that record opening of pill bottles, drug levels in plasma, blood cells, and/or hair). There is, as yet, no "gold standard" for measuring true pill-taking practices, but these studies will evaluate the correlation between various measures of pill adherence, and ultimately, whether any of these measures is highly correlated with PrEP efficacy.

All studies are also assessing sexual and drug-use practices during the course of the trial. Concern has been raised that persons may increase their risk practices if they have access to effective prevention strategies; this change in risk behavior may be due to a reduction in self-imposed limits to avoid risk (behavioral disinhibition) or decreasing perception of risk (risk compensation) with the availability of the new prevention tool $[15,16]$. The CDC-sponsored US trial of oral daily tenofovir in MSM will directly assess the potential for a change in sexual risk practices through its unique study design: half of the participants are randomized to take a daily pill (tenofovir or placebo) upon enrollment, while the other half are randomly assigned to wait 9 months before starting their daily study pill (Fig. 1). This allows a direct comparison of risk practices while men are or are not taking a daily pill, and may provide an early indication if there is likely to be substantial risk compensation. However, because participants in this trial know the efficacy of PrEP is not yet proven, and that they may be receiving placebo, this measure may under-estimate risk compensation that could occur if PrEP is found to be efficacious, and if persons are to take PrEP outside the context of a placebo-controlled trial.

Most of these studies are also powered to evaluate PrEP efficacy. Most are evaluating a single daily oral regimen, although the Partners in Prevention trial will evaluate both daily oral tenofovir versus Truvada ${ }^{\circledR}$, and the VOICE trial 


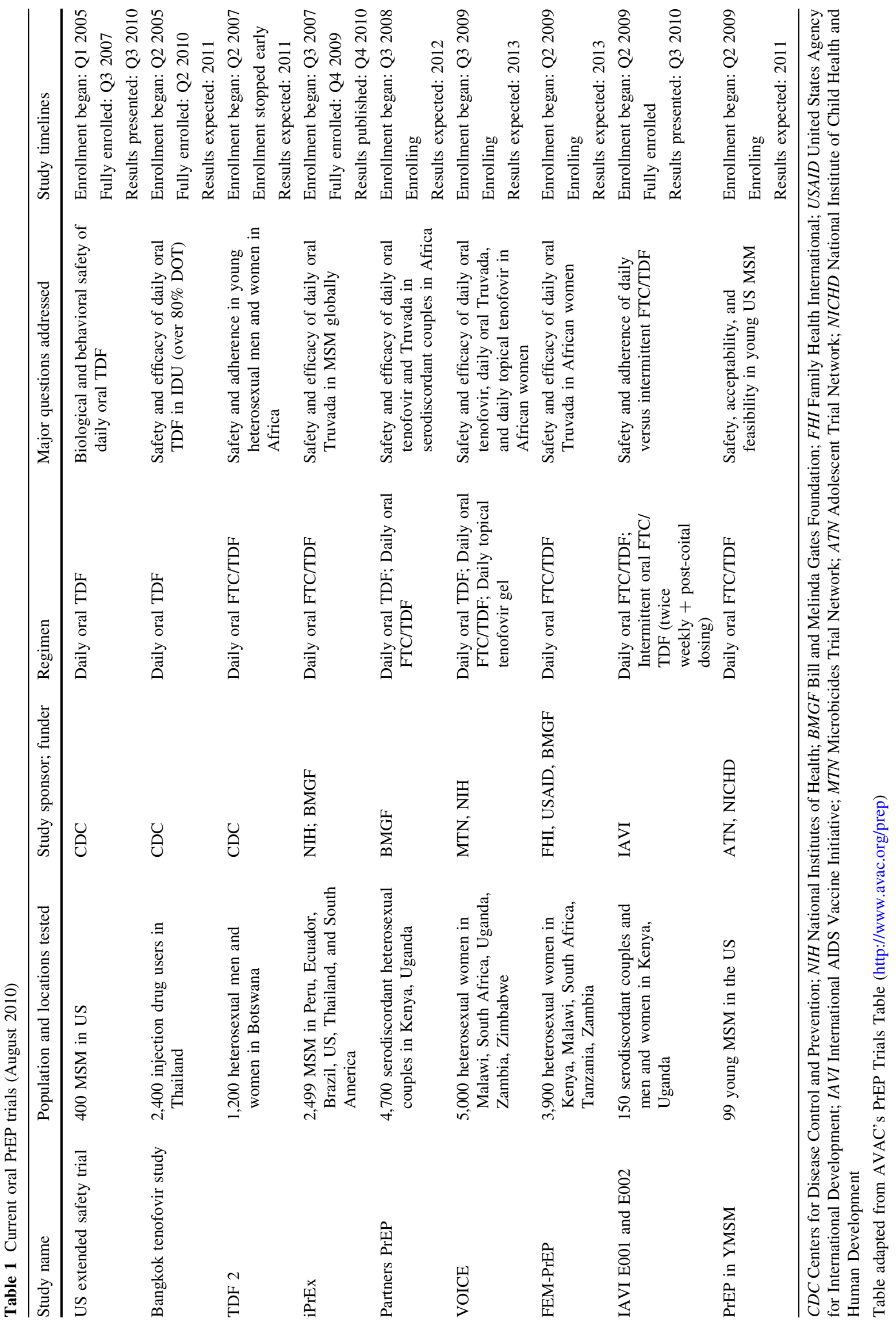




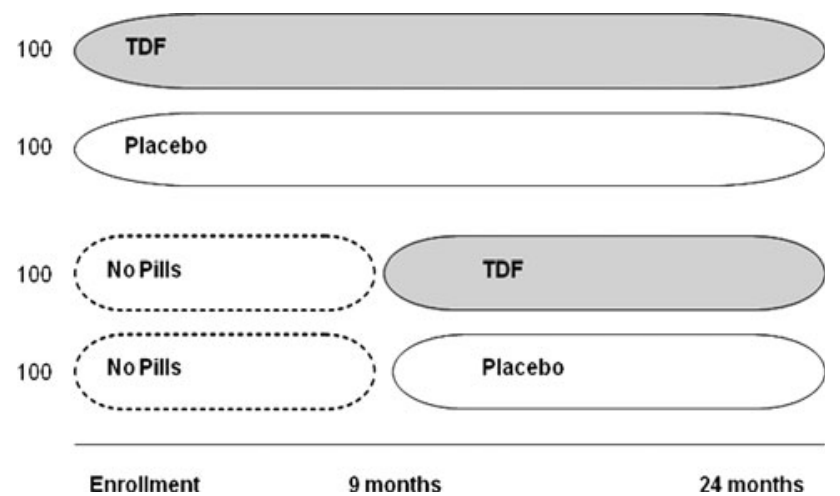

Fig. 1 Study design of US CDC tenofovir (TDF) study

will evaluate daily tenofovir, Truvada ${ }^{\circledR}$, and daily vaginal tenofovir. While neither of these multi-arm trials is powered for a direct head-to-head comparison of regimens, both will provide comparative efficacy data within the same trial. These studies will also provide preliminary data on impact on HIV viral load setpoint, CD4 cell counts, and patterns of HIV resistance among seroconverters who become infected while taking PrEP.

\section{Lessons Learned and Future Directions}

\section{Adherence is Critical}

Optimizing adherence and its measurement in prevention clinical trials is critical for trial outcomes and interpretation. The CAPRISA 004 study of tenofovir vaginal gel provided an important proof of concept that antiretroviral agents can prevent HIV acquisition [3]. However, the trial also demonstrated the importance of adherence in ultimate PrEP effectiveness: self-reported high adherers $(>80 \%$ adherence as measured by returned gel applicators) had a significant protective effect (54\% effectiveness, $95 \%$ confidence interval (CI) 4-80\%), while low adherers $(<50 \%$ adherence) had no significant protection (28\% effectiveness, 95\% CI 40-64\%). Data from the iPrEx trial of oral daily Truvada ${ }^{\circledR}$ in MSM is not yet available at the time of this writing. However, analyses are planned to evaluate the effect of adherence (as measured both by self-report and drug levels) on PrEP efficacy.

One challenge to interpreting efficacy results is the veracity of self-reported measures, where over-reporting may commonly occur. In the Carraguard vaginal microbicide trial, for instance, adherence to prescribed microbicide use was $96 \%$ via self-report but only $42 \%$ on the basis of applicator testing [17]. Data from a sub-study of the USbased MSM safety trial found relatively poor correlation between various adherence and drug exposure measures [18]. Many factors may be responsible for these discrepancies, including misunderstanding of study questions, memory lapses, social desirability bias, or differences in drug absorption, metabolism, or penetration into various tissues. Adherence and drug exposure measures will be most useful if they can reproducibly define patterns or thresholds of pill-taking required for maximum effectiveness, and point to mechanisms by which effectiveness may be impaired. Strategies to promote pill use in PrEP trials and to facilitate accurate reporting of product use are currently being explored [19].

Only limited data to date have been presented on adherence patterns in oral PrEP trials. Preliminary analysis from a comparison of daily versus fixed plus post-coital dosing in Kenya and Uganda suggested that adherence to daily and fixed-dose regimens were relatively high and better than post-coital dosing, although accurate measurement of post-coital pill use may have been an issue [2]. It is not yet known what schedule of PrEP dosing will be most effective, nor what level of adherence will be required for maximum tolerability and efficacy.

\section{Importance of Community Engagement in All Stages of Research}

Early planned PrEP trials (efficacy trials in Cambodia, Cameroon, Malawi, and three West African sites) were not launched or were stopped prematurely because of ethical, political, and logistical issues raised by community members, advocates, government officials, and clinical trial sponsors [20]. Difficult as these situations were for all parties involved, the resulting discussions that arose as a result of these challenges strengthened the next generation of trials, which included full involvement of community members, support of government officials, and strong oversight of clinical study operations. Community consultation must start early and involve multiple community groups, which may have distinct perspectives and interests. These engagement efforts should promote communication between researchers and community as well as among different community groups through transparent discussions [21]. UNAIDS has recently issued a revised version of good participatory practice (GPP) for biomedical HIV prevention trials [22]; this document highlights the importance of stakeholder engagement at each stage of the research life-cycle.

Attention to Trial Planning and Conduct is Paramount

In addition to the importance of community engagement, the successful conduct of PrEP research requires great attention to the mechanics of clinical trial implementation. PrEP trials are highly complex, and successful conduct of PrEP trials depends on talented investigators with access to 
appropriate populations to achieve rapid accrual rates, adequate seroincidence in the context of best prevention strategies, and adherence to protocol requirements. PrEP clinical trials have faced numerous challenges on these fronts. For example, the US PrEP trial among MSM experienced slower than projected enrollment; important lessons learned included the importance of centrally coordinated recruitment strategies and campaigns, and the need for streamlined regulatory approvals and support for innovative strategies, including use of the Internet [23]. Lower seroincidence was observed in both the West African PrEP study [24] and CDC Botswana trial [25], but may have been due to overall secular trends in the community, inaccurate pre-trial estimates of HIV incidence, and intensive prevention services provided in the trial. Adherence to protocol requirements is also critical. Missed visits and pregnancy were primary reasons for time off study drug in the West African PrEP study. Low retention in the CDC Botswana PrEP study contributed to the premature closure of that trial. Ideally, plans to achieve rapid enrollment, adequate HIV seroincidence, and excellent retention rates in trials should begin prior to study launch, and adequate resources should be in place to address difficulties.

\section{Many Questions Will Remain After the First Round of PrEP Studies}

Even if all of the first and second generation PrEP studies are conducted with excellent adherence to study procedures, many questions will remain after results are available (Table 2). Data to date suggest that oral tenofovir is relatively well-tolerated in HIV-negative individuals, with no substantial safety concerns after up to 12 months of use in women in West Africa [24] and up to 24 months in MSM in the US [1]. However, only preliminary safety analyses were presented on the US MSM safety trial; additional analyses, including studies of the effect of daily tenofovir or Truvada ${ }^{\circledR}$ on bone mineral density have not yet been completed. In addition, safety data have not yet been reported for other populations, including IDU and youth, and are not collected comprehensively in pregnant or lactating women, or in persons with underlying medical conditions. Also, the long-term safety of ongoing use of antiretrovirals for prevention is unknown.

All PrEP efficacy studies will measure drug resistance in breakthrough infections. However, numbers of infections on PrEP may be small, and pooling data across PrEP studies will help identify resistance issues. All current studies focus on tenofovir-based regimens; there is interest in developing other antiretroviral agents for PrEP to improve on the safety and/or efficacy profile, or to reduce the possibility of drug resistance.
Some of the most substantive questions will need to be addressed if PrEP is found to be efficacious in clinical trials. Modeling exercises suggest that PrEP could substantially reduce new infections at a population level, but that efficacy could suffer if risk practices also increase [26, 27]. If PrEP is found to be efficacious, additional studies to measure the effect of knowledge of PrEP efficacy on risk behavior and adherence will be needed. PrEP impact may be highest if delivered to those at highest risk of acquiring HIV. Several studies have surveyed MSM about their interest in taking PrEP if found to be efficacious, and these suggest that men with substantial risk may be willing to take PrEP [28-30]. However, whether and how these men might use PrEP is not yet known. Substantial work is required to determine how best to deliver PrEP to various populations in various settings [31]. PrEP delivery will require coordination of different PrEP delivery components, including outreach, screening, drug prescribing and delivery, HIV and additional safety testing, and interventions to sustain pill use and behavioral risk reduction over the long term [32]. In addition to consideration of various political, legal, and cultural issues, questions remain about the frequency and intensity of clinical monitoring and HIV testing, how best to identify and engage high-risk populations, methods of reimbursement, and the types of clinical or community-based facilities that may be best equipped to deliver PrEP in various settings.

\section{Combination Prevention Strategies}

No infectious disease has been eliminated through antimicrobials alone; control of the HIV epidemic is most likely to come through a combination of biomedical and behavioral interventions [33]. For example, the 50\% reduction in mortality from coronary heart disease from 1980 to 2000 in the US was achieved through a relatively equal contribution of modification of risk factors (e.g., decreasing prevalence of smoking) and biomedical interventions (e.g., interventional and pharmacologic therapies) [34]. Understanding the factors leading to epidemic spread, as reported in Anderson and May's classic article [35], suggests that combination interventions that target different mechanisms of an epidemic may be synergistic at a population level. For example, Hallett modeled the impact of combining widespread male circumcision with a behavior change program in South Africa [36]. In this model, widespread male circumcision $(90 \%$ coverage) alone would lead to an approximate $1 / 3$ reduction in HIV incidence at a population level; a behavior change program with a $30 \%$ reduction in partner change rate and $30 \%$ increase in condom use would lead to an approximate $2 / 3$ reduction in HIV incidence rates. However, if male 
Table 2 Data available from current PrEP trials, and areas for future studies

\begin{tabular}{|c|c|c|}
\hline & Data provided by current trials & Gaps to be addressed in future trials \\
\hline \multirow{6}{*}{$\begin{array}{l}\text { Biological safety (frequency } \\
\text { of adverse events, including } \\
\text { renal, hepatic, bone, } \\
\text { and metabolic toxicities) }\end{array}$} & Clinical safety of daily oral TDF in MSM, IDU & $\begin{array}{l}\text { Safety of longer term use (>several years) in all } \\
\text { populations }\end{array}$ \\
\hline & $\begin{array}{l}\text { Safety of FTC/TDF in MSM, heterosexual men and } \\
\text { women and serodiscordant couples in Africa }\end{array}$ & Safety of use in pregnancy or breastfeeding \\
\hline & $\begin{array}{l}\text { Safety of intermittent FTC/TDF in serodiscordant } \\
\text { couples and men and women in Africa }\end{array}$ & $\begin{array}{l}\text { Safety in persons with chronic disease (renal, } \\
\text { hepatic) }\end{array}$ \\
\hline & $\begin{array}{l}\text { Safety of daily vaginal tenofovir gel in African } \\
\text { women }\end{array}$ & Optimal frequency of safety monitoring \\
\hline & & Safety of other medications for PrEP \\
\hline & & Safety of topical tenofovir gel for rectal use \\
\hline \multirow[t]{6}{*}{ Efficacy } & $\begin{array}{l}\text { Efficacy of daily oral FTC/TDF in MSM, } \\
\text { heterosexual men and women in Africa, and } \\
\text { serodiscordant couples }\end{array}$ & $\begin{array}{l}\text { Comparative efficacy of intermittent PrEP versus } \\
\text { daily PrEP }\end{array}$ \\
\hline & Efficacy of daily oral TDF in IDU & $\begin{array}{l}\text { Efficacy of other medications for PrEP and other } \\
\text { routes of administration }\end{array}$ \\
\hline & Efficacy of daily vaginal tenofovir gel & $\begin{array}{l}\text { Mechanisms for differences (if found) in efficacy } \\
\text { results between different trials }\end{array}$ \\
\hline & $\begin{array}{l}\text { Efficacy for different routes of exposure, including } \\
\text { anal/penile (MSM), penile/vaginal (heterosexual } \\
\text { men and women), and parenteral (IDU) }\end{array}$ & \\
\hline & $\begin{array}{l}\text { Effect on HIV viral load set point and CD4 among } \\
\text { seroconverters }\end{array}$ & \\
\hline & $\begin{array}{l}\text { Validation of non-human primate models of PrEP } \\
\text { efficacy }\end{array}$ & \\
\hline \multirow[t]{2}{*}{ Adherence } & $\begin{array}{l}\text { Rates of pill or gel use based on multiple adherence } \\
\text { measures within a clinical trial }\end{array}$ & $\begin{array}{l}\text { Pill or gel use rates when efficacy of PrEP is known } \\
\text { and PrEP is taken outside of a clinical trial context }\end{array}$ \\
\hline & $\begin{array}{l}\text { Correlation between different adherence and drug } \\
\text { exposure measures }\end{array}$ & $\begin{array}{l}\text { Reproducible, easily obtained measures of drug } \\
\text { exposure that can be used in clinical practice }\end{array}$ \\
\hline Behavior & $\begin{array}{l}\text { Effect of pill-taking on risk behavior in multiple } \\
\text { populations participating in double-blind, placebo- } \\
\text { controlled studies }\end{array}$ & $\begin{array}{l}\text { Effect of pill-taking on risk behavior when efficacy } \\
\text { is known and PrEP is provided outside of a clinical } \\
\text { trial context }\end{array}$ \\
\hline \multirow[t]{2}{*}{ Resistance } & $\begin{array}{l}\text { Preliminary data on resistance patterns seen in } \\
\text { seroconverters }\end{array}$ & $\begin{array}{l}\text { Rates of drug resistance with expanded PrEP use in } \\
\text { the community }\end{array}$ \\
\hline & & $\begin{array}{l}\text { Optimal frequency of HIV testing to minimize } \\
\text { resistance but increase feasibility of PrEP delivery }\end{array}$ \\
\hline
\end{tabular}

circumcision were combined with a behavior change program, HIV incidence would be virtually eliminated.

Similar to topically-applied PrEP, which was recently shown to be $39 \%$ effective in reducing HIV infections in African women [3], oral PrEP is unlikely to be $100 \%$ effective. PrEP would therefore need to be combined with other prevention interventions to have the biggest impact. Other articles in this issue of AIDS and Behavior review other types of interventions that may be useful for controlling the HIV epidemic in MSM, and if efficacious, may be combined with PrEP to increase the protective effect. In particular, it will be important to test interventions that could be coupled with PrEP to minimize risk compensation and maximize adherence in the setting of known efficacy and implementation outside of clinical trials. Other behavioral interventions, treatment of substance abuse, rectal microbicides, increased HIV testing and disclosure, and programs to promote the sexual health and overall well-being of MSM may all play an important role in control of the HIV epidemic in MSM. Combination prevention interventions will need to be feasible to deliver and attractive to diverse MSM populations. The Prevention Umbrella for MSM in the Americas (PUMA) is an R01funded planning project to evaluate how best to combine and deliver a package of interventions to have the biggest impact on driving down HIV infection rates in MSM.

The next few years will tell us whether PrEP can be efficacious and deliverable in diverse populations of MSM worldwide. Future efforts will focus on maximizing the effectiveness and cost-effectiveness of PrEP, and 
combining PrEP with behavioral and biomedical interventions to drive down HIV incidence in MSM and other populations at risk for HIV.

Open Access This article is distributed under the terms of the Creative Commons Attribution Noncommercial License which permits any noncommercial use, distribution, and reproduction in any medium, provided the original author(s) and source are credited.

\section{References}

1. Grohskopf L, Gvetadze R, Pathak S, et al. Preliminary analysis of biomedical data from the phase II clinical safety trial of tenofovir disoproxil fumarate (TDF) for HIV-1 pre-exposure prophylaxis (PrEP) among U.S. men who have sex with men (MSM). XVIII international AIDS conference; Vienna, Austria 2010 [abstract FRLBC102].

2. Mutua GN, Sanders EJ, Kamali A, et al. Safety and adherence to intermittent emtricitabine/tenofovir for HIV pre-exposure prophylaxis (PrEP) in Kenya and Uganda. XVIII international AIDS conference; Vienna, Austria 2010.

3. Karim QA, Karim SS, Frohlich JA, et al. Effectiveness and safety of tenofovir gel, an antiretroviral microbicide, for the prevention of HIV infection in women. Science. 2010;329(5996):1168-74.

4. Freedman DO. Clinical practice. Malaria prevention in short-term travelers. N Engl J Med. 2008;359(6):603-12.

5. Panel on treatment of HIV-infected pregnant women and prevention of perinatal transmission. Recommendations for use of antiretroviral drugs in pregnant HIV-1-infected women for maternal health and interventions to reduce perinatal HIV transmission in the United States. May 24, 2010. p. 1-117. http:// aidsinfo.nih.gov/ContentFiles/PerinatalGL.pdf. Accessed Sept 2010.

6. Shih CC, Kaneshima H, Rabin L, et al. Postexposure prophylaxis with zidovudine suppresses human immunodeficiency virus type 1 infection in SCID-hu mice in a time-dependent manner. J Infect Dis. 1991;163(3):625-7.

7. Van Rompay KK, Marthas ML, Ramos RA, et al. Simian immunodeficiency virus (SIV) infection of infant rhesus macaques as a model to test antiretroviral drug prophylaxis and therapy: oral $3^{\prime}$-azido- $3^{\prime}$-deoxythymidine prevents SIV infection. Antimicrob Agents Chemother. 1992;36(11):2381-6.

8. Tsai CC, Follis KE, Sabo A, et al. Prevention of SIV infection in macaques by (R)-9-(2-phosphonylmethoxypropyl)adenine. Science. 1995;270(5239):1197-9.

9. Garcia-Lerma JG, Cong ME, Mitchell J, et al. Intermittent prophylaxis with oral truvada protects macaques from rectal SHIV infection. Sci Transl Med. 2010;2(14):14ra4.

10. Cardo DM, Culver DH, Ciesielski CA, et al. A case-control study of HIV seroconversion in health care workers after percutaneous exposure. Centers for Disease Control and Prevention Needlestick Surveillance Group. N Engl J Med. 1997;337(21):1485-90.

11. Panlilio AL, Cardo DM, Grohskopf LA, Heneine W, Ross CS. Updated U.S. Public Health Service guidelines for the management of occupational exposures to HIV and recommendations for postexposure prophylaxis. MMWR Recomm Rep. 2005;54(RR-9): $1-17$.

12. Smith DK, Grohskopf LA, Black RJ, et al. Antiretroviral postexposure prophylaxis after sexual, injection-drug use, or other nonoccupational exposure to HIV in the United States: recommendations from the U.S. Department of Health and Human Services. MMWR Recomm Rep. 2005;54(RR-2):1-20.
13. Schechter M, do Lago RF, Mendelsohn AB, Moreira RI, Moulton LH, Harrison LH. Behavioral impact, acceptability, and HIV incidence among homosexual men with access to postexposure chemoprophylaxis for HIV. J Acquir Immune Defic Syndr. 2004;35(5):519-25.

14. Celum C, Buchbinder S, Donnell D, et al. Early human immunodeficiency virus (HIV) infection in the HIV network for prevention trials vaccine preparedness cohort: risk behaviors, symptoms, and early plasma and genital tract virus load. J Infect Dis. 2001;183(1):23-35.

15. Hogben M, Liddon N. Disinhibition and risk compensation: scope, definitions, and perspective. Sex Transm Dis. 2008;35(12): 1009-10.

16. Golub SA, Kowalczyk W, Weinberger CL, Parsons JT. Preexposure prophylaxis and predicted condom use among high-risk men who have sex with men. J Acquir Immune Defic Syndr. 2010; 54(5):548-55.

17. Skoler-Karpoff S, Ramjee G, Ahmed K, et al. Efficacy of Carraguard for prevention of HIV infection in women in South Africa: a randomised, double-blind, placebo-controlled trial. Lancet. 2008; 372(9654): 1977-87.

18. Liu A, Vittinghoff E, Gandhi M, et al. Validating measures of tenofovir drug exposure in a US pre-exposure prophylaxis trial. 17th conference on retroviruses and opportunistic infections; San Francisco, CA 2010 [abstract 86].

19. Amico KR. Developing an innovative approach to adherence counseling and assessment in a pre-exposure prophylaxis (PrEP) trial: next step counseling and neutral assessment in the iPrEx study. Fifth international conference on HIV treatment adherence; Miami, FL 2010 [abstract 62253].

20. Mills E. Tenofovir trials raise ethical issues. HIV AIDS Policy Law Rev. 2005;2:31-2.

21. Goicochea LP, McConnell J, Lama J, et al. Finding the community in 'community consultation' to prepare for biomedical HIV prevention trials. Thirteenth conference on retroviruses and opportunistic infection. Denver, CO 2006 [abstract 898].

22. UNAIDS/AVAC. Good participatory practice guidelines for biomedical HIV prevention trials. 2nd ed. Geneva: Joint United Nations Programme on HIV/AIDS (UNAIDS) 2010. p. 80.

23. Grohskopf L, Gvetadze R, Pathak S, et al. Recruitment challenges and strategies in the phase II extended safety trial of tenofovir (TDF) HIV chemoprophylaxis in three US cities among men who have sex with men (MSM). XVIII international AIDS conference; Vienna, Austria 2010 [abstract THPE0242].

24. Peterson L, Taylor D, Roddy R, et al. Tenofovir disoproxil fumarate for prevention of HIV infection in women: a phase 2, double-blind, randomized, placebo-controlled trial. PLoS Clin Trials. 2007;2(5):e27.

25. CDC. Status of the Botswana TDF2 study of pre-exposure prophylaxis for HIV prevention 2009. www.cdc.gov/hiv/prep/resources/fact sheets/botswanatdf2.htm. Accessed Sept 2010.

26. Abbas UL, Anderson RM, Mellors JW. Potential impact of antiretroviral chemoprophylaxis on HIV-1 transmission in resource-limited settings. PLoS ONE. 2007;2(9):e875.

27. Supervie V, Garcia-Lerma JG, Heneine W, Blower S. HIV, transmitted drug resistance, and the paradox of preexposure prophylaxis. Proc Natl Acad Sci USA. 2010;107(27):12381-6.

28. Liu AY, Kittredge PV, Vittinghoff E, et al. Limited knowledge and use of HIV post- and pre-exposure prophylaxis among gay and bisexual men. J Acquir Immune Defic Syndr. 2008;47(2): 241-7.

29. Mimiaga MJ, Case P, Johnson CV, Safren SA, Mayer KH. Preexposure antiretroviral prophylaxis attitudes in high-risk Boston area men who report having sex with men: limited knowledge and experience but potential for increased utilization after education. J Acquir Immune Defic Syndr. 2009;50(1):77-83. 
30. Poynten IM, Jin F, Prestage GP, Kaldor JM, Imrie J, Grulich AE. Attitudes towards new HIV biomedical prevention technologies among a cohort of HIV-negative gay men in Sydney, Australia. HIV Med. 2010;11(4):282-8.

31. Kim SC, Becker S, Dieffenbach $\mathrm{C}$, et al. Planning for preexposure prophylaxis to prevent HIV transmission: challenges and opportunities. J Int AIDS Soc. 2010;13(1):24.

32. Underhill K, Operario D, Mimiaga MJ, Skeer MR, Mayer KH. Implementation science of pre-exposure prophylaxis: preparing for public use. Curr HIV/AIDS Rep. 2010;7(4):210-9.

33. Coates TJ, Richter L, Caceres C. Behavioural strategies to reduce HIV transmission: how to make them work better. Lancet. 2008;372(9639):669-84.
34. Ford ES, Ajani UA, Croft JB, et al. Explaining the decrease in U.S. deaths from coronary disease, 1980-2000. N Engl J Med. 2007;356(23):2388-98.

35. Anderson RM, May RM, Boily MC, Garnett GP, Rowley JT. The spread of HIV-1 in Africa: sexual contact patterns and the predicted demographic impact of AIDS. Nature. 1991;352(6336): 581-9.

36. Hallett TB, Singh K, Smith JA, White RG, Abu-Raddad LJ, Garnett GP. Understanding the impact of male circumcision interventions on the spread of HIV in southern Africa. PLoS ONE. 2008;3(5):e2212. 